\title{
Introduction to the Scanning Electron Microscope
}

\author{
David C Joy \\ University of Tennessee, Knoxville, TN 37996-0840 and \\ Oak Ridge National Laboratory, Oak Ridge, TN 37831-6064
}

The scanning electron microscope (SEM), a member of the same family of imaging devices as FAX machines and televisions. is the most widely used of all electron beam tools with some 40,000 units distributed worldwide ${ }^{[1]}$. The SEM employs a focussed beam of electrons, with energies typically in the range from a few hundred $\mathrm{eV}$ to about $30 \mathrm{keV}$, which is rastered across the surface of a sample in a rectangular scan pattern. Signals emitted under this electron irradiation are collected, amplified, and then used to modulate the brightness of a suitable display device which is being scanned in synchronism with probe beam. This arrangement has several important benefits; (1) the magnification, which is the ratio of the areas scanned on the display device and on the sample, is obtained geometrically and so does not rely on lenses; (2) any emission from the sample that can be collected can be used to form an image; (3) multiple images using different signals can be collected simultaneously; (4) because the signal is acquired sequentially it can be processed and enhanced before it is displayed. The resolution of the SEM cannot be better than the dimension of the pixels used to display the image, and this limitation controls the imaging performance for all magnifications lower than about 20,000x. At higher magnifications, however, the resolution may be determined by other considerations including the type of signal that is being employed, the signal to noise ratio, the physical size of the electron probe, and the width of the electron-solid interaction. Effects such as sample charging and beam induced damage also, increasingly, influence performance and the choice of operating conditions.

The most widely employed modes of use are called, mostly for historical reasons, secondary electron (SE) and backscattered electron (BSE) imaging. Secondary electrons, defined as being those with energies between 0 and $50 \mathrm{eV}$, are easy to collect with good efficiency and can be employed over a very wide range of incident beam energies. The SE image offers surface specific, high resolution, topographic detail which can readily be interpreted using a simple optical analogy. In addition it may contain information about the chemistry, and the electrical 
and magnetic state of the sample. Backscattered electrons constitute all the other electrons emitted from the specimen. BSE detectors are typically larger and less efficient than the SE detector especially at low beam energies and this has tended to restrict the popularity of the mode. Contrast in the BSE image is directly sensitive to the atomic number of the sample, as well as to its magnetic and crystallographic nature and, in general, comes from the bulk rather than from the surface of the sample. Other commonly employed imaging and analytical modes include the use of emitted light (cathodo-luminescence), signals generated by electron-hole pairs (electron beam induced charge), and fluorescent X-rays.

Although the commercial SEM has now been under continuous development for 40 years, important new advances in instrumentation continue to be made. Of particular significance are the new high brightness electron sources, such as Schottky and cold field emitters, which offer higher currents into smaller diameter probes. Major advances in the design of lenses have also been achieved including the first applications of hardware which can minimize or eliminate the electron-optical aberrations which degrade probe size and limit the probe current. New types of detector configurations, which are designed to be sensitive to the angular and/or energy distribution of the electrons, provide enhanced contrast and new methodologies of image formation. Finally, innovative vacuum designs have resulted in the so-called environmental (ESEM) or variable pressure (VPSEM) instruments which permit the sample to be imaged without need for it to be in high vacuum and offer unique advantages for the observation of wet, dirty, or non-conducting samples. However, major problems also remain unresolved. The depth of field of high performance SEMs when operated at high magnification is now measured in nanometers rather than in micrometers, and charging, and radiation damage, represent fundamental barriers to the application of the SEM to many specimens of interest ${ }^{[2]}$.

\section{Reference}

(1) J I Goldstein et al, Scanning Electron Microscopy and X-ray Microanalysis, ( $3^{\text {rd }}$ edition), Kluewer Press:New York, 2003

(2) Oak Ridge National Laboratory is operated by UT-Batelle LLC, for the USDOE under contract DE-AC05-00OR22725 